\title{
How do nematodes transfer phosphorylcholine to carbohydrates?
}

\author{
William Harnett, Justyna Rzepecka and Katrina M. Houston
}

Strathclyde Institute of Pharmacy and Biomedical Sciences, The John Arbuthnott Building, 27 Taylor Street, Glasgow G4 0NR, UK

\begin{abstract}
An unusual aspect of the biology of nematodes is the attachment of phosphorylcholine (PC) to carbohydrate. The attachment appears to play an important role in nematode development and, in some parasitic species, in immunomodulation. This article considers the nature of the biosynthetic pathway of nematode PC-containing glycoconjugates and, in particular, the identity of the final component in the pathway - the enzyme that transfers PC to carbohydrate (the 'PC transferase'). We offer the opinion that the PC transferase could be a member of the fukutin family (fukutin refers to the mutated gene product that causes Fukuyama congenital muscular dystrophy), a group of enzymes with apparent phosphoryl-ligand transferase activity that are found in organisms ranging from bacteria to humans.
\end{abstract}

The discovery of phosphorylcholine-glycoconjugates in nematodes

Eukaryotes including nematodes contain abundant levels of phosphorylcholine (PC). This PC is usually detected as the polar head group of lipids such as phosphatidylcholine and sphingomyelin; however, during the $1970 \mathrm{~s}$ (Ref. [1]) and $1980 \mathrm{~s}$ (Ref. [2]) evidence (based on simple chemical and immunochemical procedures) began to emerge from studies on parasitic nematodes that PC was present on carbohydrate-containing molecules. In the late $1980 \mathrm{~s}$ we showed (using biosynthetic radiolabelling procedures) that it was possible to incorporate both glucosamine and choline into a major PC excretory-secretory (ES) molecule with a molecular mass of $\sim 62 \mathrm{kDa}$ (referred to as ES-62), a secreted protein of the filarial nematode Acanthocheilonema viteae [3]. Furthermore, during the $1990 \mathrm{~s}$ it was demonstrated, by the use of enzymes that cleave carbohydrate [4], and also by employing inhibitors of glycosylation initiation and processing $[5,6]$, that the PC on ES-62 was apparently attached directly to carbohydrate and, in particular, to an N-type glycan. These studies produced the first indication of such a chemical structure and, in collaboration with Anne Dell's group at Imperial College, we subsequently confirmed its existence by fast atom bombardment mass spectroscopy (FABMS) [7]. Since then, PC$\mathrm{N}$-glycans have been detected in other filarial nematodes [8], in parasitic gastrointestinal nematodes (for example, Ref. [9]) and also in the free-living nematode Caenorhabditis elegans [10]. Moreover, PC has been found covalently attached to carbohydrate in nematode glycosphingolipids [11].

Corresponding author: Harnett, W. (w.harnett@strath.ac.uk).
The probable conservation of two forms of PC-containing glycoconjugate across the entire phylum, allied to an apparent abundance and widespread anatomical distribution of such molecules (reviewed in Ref. [12]), argues that the addition of PC to carbohydrate has essential biological functions in nematodes. Consistent with this, over the past 15 years our research group has demonstrated that the PC moiety of ES-62 has powerful immunomodulatory properties. It is able to interact directly with cells of the immune system including lymphocytes, dendritic cells and macrophages, the net effect being to polarise the immune response towards an anti-inflammatory phenotype that is believed to be conducive to parasite survival (reviewed in Ref. [13]). Similar properties have been found in PC-containing glycoconjugates of other nematodes, for example Ascaris glycosphingolipids [14]. The function of PC-containing carbohydrates within nematodes however awaits elucidation. Clearly, they do not necessarily have an immunomodulatory role because they are found in free-living species. A clue to this conundrum might be provided by studies on bacteria. PC has been found attached to carbohydrate on teichoic acid [15] and lipoteichoic acid [16] in some of Gram-positive bacteria and to sugars of lipopolysaccharide (LPS) in certain Gramnegative species [17]. Studies on Streptococcus species indicate that the PC, possibly due to a charge effect, is essential for maintenance of cell shape and size and for promoting growth and cell division (reviewed in Ref. [18]). It is possible that internal PC-containing glycoconjugates of nematodes could play similar roles; in support, chemical inhibition of the synthesis of PC-containing glycosphingolipids by targeting enzymes upstream of that which catalyses PC addition was reported to impair embryonic development in C. elegans [19]. Also of interest, but yet to be explained in terms of nematode biology, is the recent observation that there are clear differences in the structure of PC-containing glycans in the different stages of $C$. elegans [20].

\section{What is the PC acceptor?}

Using the A. viteae-ES-62 system, we were the first to investigate the biosynthetic pathway responsible for the addition of PC to carbohydrates in nematodes. ES-62 is synthesised by highly metabolically active cells that subtend the oesophagus of the worm [21]. The wealth of accumulated data is consistent with the idea that PC is added to ES-62 in these cells during oligosaccharide processing (reviewed in Ref. [22]). These data are derived from pulse-chase radiolabelling experiments, experiments 


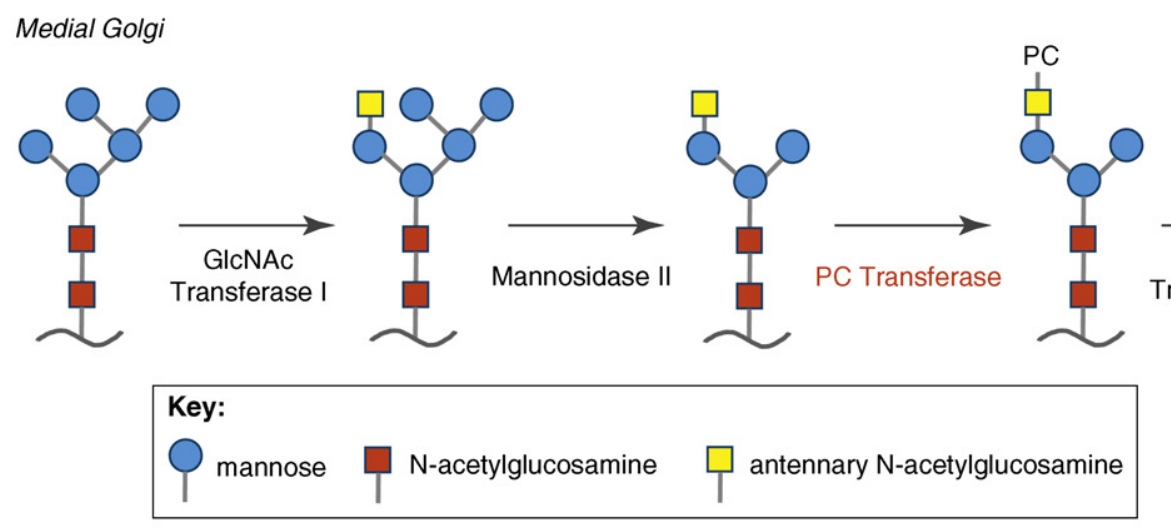

$\overline{\text { TRENDS in Parasitology }}$

Figure 1. Transfer of phosphorylcholine (PC) to N-glycans on ES-62 occurs during oligosaccharide processing in the medial Golgi. PC is first transferred to the antennary Nacetylglucosamine (GICNAc) residue that is added by GIcNAc transferase I to the 3-linked mannose of the trimannosyl core of an N-type glycan. This takes place in the medial Golgi after the glycan has been processed by mannosidase II, but prior to processing by GIcNAc transferase II.

using inhibitors of intracellular trafficking and oligosaccharide processing, and FABMS analysis of the N-type glycans to which PC is attached. Specifically, it appears likely that PC is first transferred to the antennary $N$ acetylglucosamine (GlcNAc) residue that is added by GlcNAc transferase I to the 3-linked mannose of the trimannosyl core of an N-type glycan (Figure 1). This takes place after the glycan has been processed by mannosidase II, but prior to processing by GlcNAc transferase II in the medial Golgi. However, further addition of PC to GlcNAcs might then take place after GlcNAc transferases, such as GlcNAc transferase II, act on the glycan. There are also indications that PC can be transferred to core in addition to terminal GlcNAc residues on N-type glycans [20].

In addition, other studies have suggested that, in nematodes, PC might be attached to proteins via the polypeptide backbone [23,24], and one study reported structural data suggesting that PC attachment can occur via mannose residues on $\mathrm{N}$-glycans with a pentamannosyl core structure [25]. However, as with the work on ES-62, the vast majority of studies to date have found that PC on nematode $N$-type glycans is attached via GlcNAc. Attachment is via a phosphodiester linkage at the C-6 position [10] (Figure 2) and this is also the case for glycosphingolipids [26]. Thus, in general, the acceptor for the PC transferase appears to be simply the C6-position of a GlcNAc residue, with transfer taking place regardless of the nature of glycoconjugate. However, studies by Cipollo and colleagues [27] employing a variety of $\mathrm{N}$-glycans and an in vitro $\mathrm{PC}$ transferase assay utilising a $C$. elegans microsomal extract revealed that the nature of the GlcNAc-containing molecule influences the efficiency of PC transfer. In particular it was noticeable that several glycans containing terminal GlcNAc were not substituted by PC, thereby raising the possibility that the nature and position of other sugars of the glycan might influence whether transfer takes place. This could be investigated further by manipulating GlcNAc-containing glycan composition and structure and establishing the effects on PC transfer.

\section{What is the PC donor?}

Radiolabelling experiments indicate that the PC transferred to ES-62 is derived from exogenous choline, and attachment is inhibited when worms are incubated in the presence of the choline kinase inhibitor, hemicholinium-3 (HC-3) (Ref. [28]). This led us to consider that the PC donor for ES-62 might be a component of the Kennedy Pathway of phospholipid biosynthesis, specifically CDP-choline, phosphatidylcholine or sphingomyelin. Pulse-chase experiments with adult $A$. viteae employing $\left[{ }^{3} \mathrm{H}\right]$-choline as

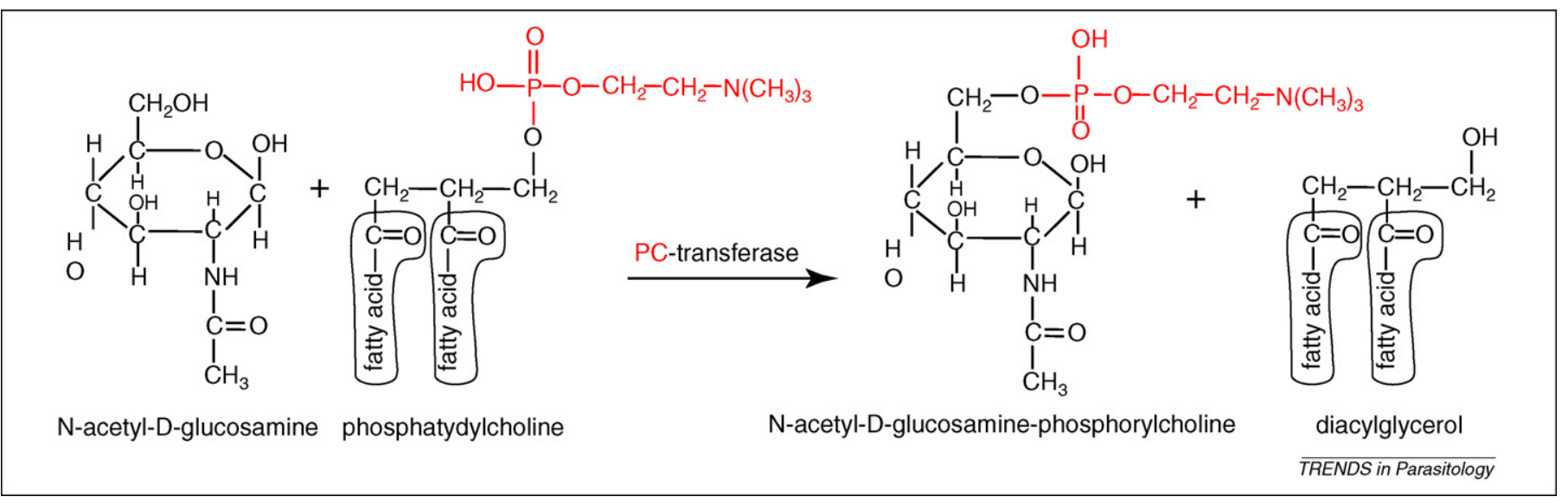

Figure 2. Model for transfer of PC to carbohydrate in nematodes. PC from the donor, phosphatidylcholine, is transferred by an unknown enzyme, the PC-transferase, to Nacetylglucosamine (GIcNAc) on N-glycans and glycosphingolipids. Attachment is via a phosphodiester linkage at the C-6 position of GlcNAc. Diacylglycerol is likely to be produced as a consequence of utilization of phosphatidylcholine as the PC donor. 
the radiolabel ruled out sphingomyelin because it was labelled 20-30 minutes after ES-62 (Ref. [29]). Although both CDP-choline and phosphatidylcholine were labelled before ES-62, using established methods of pulse-chase and enzyme inhibition we were unable to conclude that these were donors [28,29]. Cipollo and colleagues subsequently demonstrated that phosphatidylcholine, but not CDP-choline, could act as a PC donor in the in vitro $\mathrm{PC}$ transferase assay using a C. elegans extract mentioned earlier [27]. Thus, at this stage, phosphatidylcholine appears to be the donor for transfer of PC to sugars in C. elegans at least (Figure 2).

\section{What is the PC transferase?}

As mentioned earlier, PC appears to be transferred to ES62 during oligosaccharide processing in the medial Golgi [6]. The PC transferase is likely to be membrane-bound because, unlike the ER, there is no evidence for a family of soluble resident proteins within the Golgi lumen (reviewed in Ref. [30]). This could be analogous to other enzymes in the local environment (e.g. glycosyltransferases) where the enzyme is a type II transmembrane protein with a short $\mathrm{N}$ terminal cytoplasmic domain, a single transmembrane segment, and a large C-terminal domain containing the active site within the Golgi lumen (reviewed in Ref. [31]). Various search programs can be applied to the C. elegans genome to predict the intracellular locations of proteins; although laborious, it would be possible to investigate all genes considered to fall into the Golgi-located type II transmembrane protein category for PC transferase activity. However, we cannot be absolutely certain of a membrane-bound location, and in any case the probable characteristics of the PC transferase described above suggest that it is perhaps most likely to resemble a glycosyltransferase or an enzyme involved in choline metabolism such as sphingomyelin synthase (that, like the unknown PC transferase, uses phosphatidylcholine as a $\mathrm{PC}$ donor). Analysis of the C. elegans genome indicates the existence of multiple genes that appear to fall into either category, but there is one additional group of genes that represents a particularly attractive starting point in the search for a PC transferase. The gene encoding the enzyme responsible for transfer of $\mathrm{PC}$ to carbohydrate in bacteria, LicD, has been defined [32], and this appears to be a member of the fukutin family (named after the mutated gene product that causes Fukuyama congenital muscular dystrophy and whose function remains unknown) [33]

\section{Box 1. The fukutin protein family}

Fukutin is a protein associated with Fukuyama congenital muscular dystrophy [33]. Specifically, mutations within this protein result in the phenotypic changes associated with brain malformation that are observed in this disease. The protein contains a signal peptide and has been localized to the Golgi and secretory granules. It is predicted to be an enzyme that modifies glycoproteins or glycolipids on the surface of cells.

The fukutin family can be defined by a motif with the sequence G[TS]hhGhhx4hhxaxxDxD in which ' $h$ ' is a hydrophobic amino acid, ' $a$ ' an aromatic amino acid, and ' $x$ ' is any amino acid [34]. The LicD gene products of Streptococcus pneumoniae [35] and Haemophilus influenzae [32] appear to transfer phosphorylcholine to carbohydrate on teichoic acid and lipopolysaccharide respectively. Similarly, another member of the family, the yeast protein MNN4, transfers mannosyl phosphate from a GDP-mannose donor to glycans [36]. These studies, together with the sequence conservation pattern, prompted Aravind and Koonin to suggest that fukutin family proteins are phosphoryl-ligand transferases [34]. They further comment that the presence of the DxD motif, and also a distal aspartic acid residue present in most family members, suggests that the proteins coordinate a divalent cation analogous to a number of nucleotidyltransferases.

(Box 1). Members of this recently discovered family appear to resemble glycosyltransferases [37], consisting of phosphoryl-ligand transferases that modify carbohydrate on glycoproteins and glycolipids (reviewed in Ref. [34]). Furthermore, murine fukutin was recently shown to be a type II transmembrane protein located in the medial Golgi [38]. The C. elegans genome contains a number of 'fukutin-related' genes (Figure 3) and it will be interesting to establish whether worms that have these genes knocked out or reduced by RNAi demonstrate reduced PC transferase activity. However, it should be noted that, using RNAi, a reduction in $\mathrm{PC}$ addition was observed when the genes F22E10.5 and FC8C6.2 were targeted [40]. These might indeed encode enzymes that transfer PC to carbohydrate, but we predict (based on sequence homology) that they are more likely to encode the enzymes responsible for the synthesis of phosphatidylcholine (DAG:choline phosphotransferase) and CDP-choline (CTP:phosphocholine cytidylyltransferase) respectively. The reason that their inactivation results in reduced PC transfer to glycans is thus almost certainly because of reductions in PC donor levels $[27,29]$. Hence, the only way to be certain that nematode fukutin-related genes truly encode enzymes that transfer PC to carbohydrate will be to clone and express them, and then test for PC transferase enzyme activity using assays as described by Cipollo and colleagues [27].

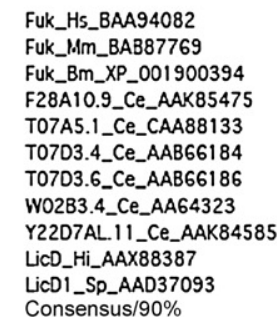

Fuk_Hs_BAA94082

Fuk_Bm_XP_001900394 F28A10.9_Ce_AAK85475 T0703.4_Ce_AAB66184 TO7D3.6_Ce_AAB66186 Y2207AL.11_Ce_AAK8458 ICD1_SP_AAD37093 Consensus $/ 90 \%$

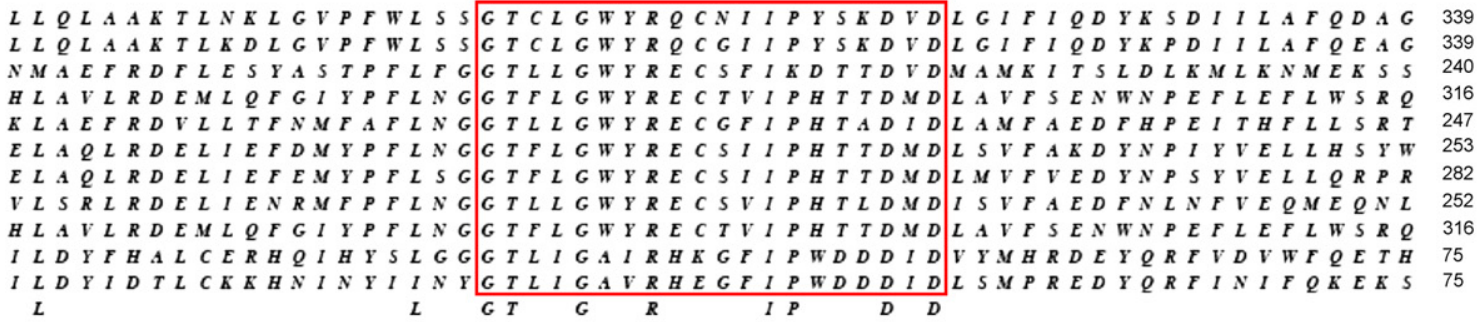

TRENDS in Parasitology

Figure 3. A multiple alignment of fukutin family proteins. The protein designations include: gene name_species abbreviation_GenBank identification number. The species abbreviations are as follows: Hm, Homo sapiens; Mm, Mus musculus; Bm, Brugia malayi; Ce, Caenorhabditis elegans; Hi, Haemophilus influenzae; Sp, Streptococcus pneumoniae. The conserved signature consisting of 20 amino acid residues is contained within the red box. 
One additional question that has to be addressed is: how many PC transferases do nematodes possess? It is possible that, in nematodes, one enzyme might transfer PC both to differently positioned GlcNAcs on N-glycans and to glycosphingolipids in the appropriate Golgi compartments. Certainly, a number of glycosyltransferases have been shown to act on both classes of glycoconjugate in vitro (reviewed in Ref. [39]) and, as mentioned earlier, the acceptor for PC is the same in both classes of molecule (C-6 position of $\beta$ linked GlcNAc [10,11]). Nevertheless, two distinct LicD gene products have been described for Haemophilus influenzae [32] (each enzyme transferring PC to a differently located sugar on LPS) and Streptococcus pneumoniae is also suspected of having two enzymes [35]. Thus, it is not inconceivable that each species of nematode could contain several enzymes that transfer PC to carbohydrate.

\section{Conclusions and future directions}

The mechanism by which nematodes transfer PC to sugars on glycoconjugates has been considered here, and data have been reviewed that give indications as to the identities of the PC donor - phosphatidylcholine, and of the PC acceptor - the $\mathrm{C} 6$ position of certain GlcNAc residues. The identity of the enzyme that transfers PC to carbohydrate remains elusive, but we offer the opinion, based mainly on analogies with PC transfer to carbohydrate in bacteria, that it could be a member of the fukutin-related family. In addition to furthering our understanding of this unique biosynthetic pathway in nematodes, given the possibility that nematode PC transferases could act as urgently sought novel drug targets, there is a strong argument for investigating this opinion experimentally using procedures as outlined above.

\section{References}

1 Pery, P. et al. (1974) Phosphorylcholine-containing components in homogenates of nematodes. Eur. J. Immunol. 4, 637-639

2 Maizels, R.M. et al. (1987) Phosphorylcholine-bearing antigens in filarial nematode parasites: analysis of somatic extracts, in vitro secretions and infection sera from Brugia malayi and B. pahangi. Parasite Immunol. 9, 49-66

3 Harnett, W. et al. (1989) Origin, kinetics of circulation and fate in vivo of the major excretory-secretory product of Acanthocheilonema viteae. Parasitology 99, 229-239

4 Harnett, W. et al. (1993) Acanthocheilonema viteae: phosphorylcholine is attached to the major excretory-secretory product via an $N$-linked glycan. Exp. Parasitol. 77, 498-502

5 Houston, K.M. and Harnett, W. (1996) Prevention of attachment of phosphorylcholine to a major excretory-secretory product of Acanthocheilonema viteae using tunicamycin. J. Parasitol. 82, 320-324

6 Houston, K.M. et al. (1997) Studies on the site and mechanism of attachment of phosphorylcholine to a filarial nematode secreted glycoprotein. J. Biol. Chem. 272, 1527-1533

7 Haslam, S.M. et al. (1997) Characterisation of the phosphorylcholinecontaining $N$-linked oligosaccharides in the excretory-secretory $62 \mathrm{kDa}$ glycoprotein of Acanthocheilonema viteae. Mol. Biochem. Parasitol. 85, 53-66

8 Haslam, S.M. et al. (1999) Structural studies of N-glycans of filarial parasites: conservation of phosphorylcholine-substituted glycans among species and discovery of novel chito-oligomers. J. Biol. Chem. 274, 20953-20960

9 Morelle, W. et al. (2000) Phosphorylcholine-containing $N$-glycans of Trichinella spiralis: identification of multiantennary lacdiNAc structures. Glycobiology 10, 941-950

10 Haslam, S.M. et al. (2002) The glycomes of Caenorhabditis elegans and other model organisms. Biochem. Soc. Symp. 69, 117-134
11 Gerdt, S.et al. (1999) Isolation, characterisation and immunolocalization of phosphorylcholine-containing glycolipids in developmental stages of Caenorhabditis elegans. Eur. J. Biochem. 266, 952-963

12 Lochnit, G. et al. (2000) Phosphorylcholine substituents in nematodes: structures, occurrence and biological implications. Biol. Chem. 381, 839-847

13 Harnett, W. and Harnett, M.M. (2006) Filarial nematode secreted product ES-62 is an anti-inflammatory agent: therapeutic potential of small molecule derivatives and ES-62 peptide mimetics. Clin. Exp. Pharmacol. Physiol. 33, 511-518

14 Deehan, M.R. et al. (2002) Immunomodulatory properties of Ascaris suum glycoshingolipids - phosphorylcholine and nonphosphorylcholine-dependent effects. Parasite Immunol. 24, 463-469

15 Brundish, D.E. and Baddiley, J. (1968) Pneumococcal C-substance: a ribotol teichoic acid containing choline phosphate. Biochem. J. 110, $573-582$

16 Briles, E.B. and Tomasz, A. (1973) Pneumococcal Forsmann antigen: a choline-containing lipoteichoic acid. J. Biol. Chem. 248, 6394-6397

17 Weiser, J.N. et al. (1997) Decoration of lipopolysaccharide with phosphorylcholine: a phase-variable characteristic of Haemophilus influenzae. Infect. Immun. 65, 943-950

18 Harnett, W. and Harnett, M.M. (1999) Phosphorylcholine: friend or foe of the immune system? Immunol. Today 20, 125-129

19 Lochnit, G. et al. (2005) Searching new targets for anthelmintic strategies: interference with glycosphingolipid biosynthesis and phosphorylcholine metabolism affects development of Caenorhabditis elegans. Int. J. Parasitol. 35, 911-923

20 Cipollo, J.F. et al. (2005) $N$-glycans of Caenorhabditis elegans are specific to developmental stages. J. Biol. Chem. 280, 26063-26072

21 Stepek, G. et al. (2002) Expression of the filarial nematode phosphorylcholine-containing glycoprotein, ES-62, is stage-specific. Parasitology 125, 155-164

22 Houston, K.M. and Harnett, W. (2004) Structure and synthesis of nematode phosphorylcholine-containing glycoconjugates. Parasitology 129, 655-662

23 Lochnit, G. et al. (2006) First identification of a phosphorylcholinesubstituted protein From Caenorhabditis elegans: isolation and characterisation of the aspartyl protease ASP-6. Biol. Chem. 387, 1487-1493

24 Grabitzki, J. et al. (2008) The PCome of Caenorhabditis elegans as a prototypic model system for parasitic nematodes: identification of phosphorylcholine-substituted proteins. Mol. Biochem. Parasitol. $161,101-111$

25 Cipollo, J.F. et al. (2002) The fine structure of Caenorhabditis elegans N-glycans. J. Biol. Chem. 277, 49143-49157

26 Lochnit, G. et al. (1998) Structural elucidation and monokine-inducing activity of two biologically active zwitterionic glycosphingolipids derived from the porcine parasitic nematode Ascaris suum. J. Biol. Chem. 273, 466-474

27 Cipollo, J.F. et al. (2004) Biosynthesis in vitro of Caenorhabditis elegans phosphorylcholine oligosaccharides. Proc. Natl. Acad. Sci. U. S. A. $101,3404-3408$

28 Houston, K.M. and Harnett, W. (1999) Mechanisms underlying the transfer of phosphorylcholine to filarial nematode glycoproteins - a possible role for choline kinase. Parasitology 118, 311-318

29 Houston, K.M. et al. (2002) Investigation of the nature of potential phosphorylcholine donors for filarial nematode glycoconjugates. $\mathrm{Mol}$. Biochem. Parasitol. 123, 55-66

30 Munro, S. (1998) Localisation of proteins to the Golgi apparatus. Trends Cell Biol. 8, 11-15

31 Breton, C. and Imberty, A. (1999) Structure/function studies of glycosyltransferases. Curr. Opin. Struct. Biol. 9, 563-571

32 Lydsenko, E. et al. (2000) The position of phosphorylcholine on the lipopolysaccharide of Haemophilus influenzae affects binding and sensitivity to C-reactive protein-mediated killing. Mol. Microbiol. 35, 234-245

33 Kobayashi, K. et al. (1998) An ancient retrotransposal insertion causes Fukuyama-type congenital muscular dystrophy. Nature 394, 388-392

34 Aravind, L. and Koonin, E.V. (1999) The fukutin protrein family predicted enzymes modifying cell surface molecules. Curr. Biol. 9, R836-R837

35 Zhang, J.R. et al. (1999) Pneumococcal LicD2 gene is involved in phosphorylcholine metabolism. Mol. Microbiol. 31, 1477-1488 
36 Jugami, Y. and Odani, T. (1999) Mannosylphosphate transfer to yeast mannan. Biochim. Biophys. Acta 1426, 335-345

37 Yuan, Y.P. et al. (1997) Secreted fringe-like signalling molecules may be glycosyltransferases. Cell 88, 9-11

38 Esapa, C.T. et al. (2002) Functional requirements for fukutin-related protein in the Golgi apparatus. Hum. Mol. Genet. 11, 3319-3331
39 Paulson, J.C. and Colley, K.J. (1989) Glycosyltransferases. Structure, localization, and control of cell type-specific glycosylation. J. Biol. Chem. 264, 17615-17618

40 Houston, K.M. et al. (2008) Gene inactivation confirms the identity of enzymes involved in nematode phosphorylcholine- $N$-glycan synthesis. Mol. Biochem. Parasitol. 157, 88-91 